OPEN ACCESS

Edited by:

Mohammad Anwar Hossain, Bangladesh Agricultural University,

Bangladesh

Reviewed by:

Gaurav Zinta

Chinese Academy of Sciences, China Chandrasekhar Kottakota, Agricultural Research

Organization-Volcani Center, Israel

*Correspondence:

Bhavanath Jha

bjha@csmcri.org

${ }^{\dagger}$ These authors have contributed equally to this work.

Specialty section:

This article was submitted to

Plant Biotechnology,

a section of the journal

Frontiers in Plant Science

Received: 17 March 2016 Accepted: 13 May 2016

Published: 02 June 2016

Citation:

Singh D, Yadav NS, Tiwari V Agarwal PK and Jha B (2016) A SNARE-Like Superfamily Protein SbSLSP from the Halophyte Salicornia brachiata Confers Salt and Drought Tolerance by Maintaining Membrane

Stability, $\mathrm{K}^{+} / \mathrm{Na}^{+}$Ratio, and Antioxidant Machinery.

Front. Plant Sci. 7:737.

do: 10.3389/fpls.2016.00737

\section{A SNARE-Like Superfamily Protein SbSLSP from the Halophyte Salicornia brachiata Confers Salt and Drought Tolerance by Maintaining Membrane Stability, $\mathrm{K}^{+} / \mathrm{Na}^{+}$Ratio, and Antioxidant Machinery}

Dinkar Singh ${ }^{1 \dagger}$, Narendra Singh Yadav ${ }^{1 \dagger}$, Vivekanand Tiwari ${ }^{1}$, Pradeep K. Agarwal ${ }^{1,2}$ and Bhavanath Jha ${ }^{1,2 *}$

${ }^{1}$ Division of Marine Biotechnology and Ecology, CSIR-Central Salt and Marine Chemicals Research Institute, Bhavnagar, India, ${ }^{2}$ Academy of Scientific and Innovative Research, CSIR, New Delhi, India

About 1000 salt-responsive ESTs were identified from an extreme halophyte Salicornia brachiata. Among these, a novel salt-inducible gene SbSLSP (Salicornia brachiata SNARE-like superfamily protein), showed up-regulation upon salinity and dehydration stress. The presence of cis-regulatory motifs related to abiotic stress in the putative promoter region supports our finding that SbSLSP gene is inducible by abiotic stress. The SbSLSP protein showed a high sequence identity to hypothetical/uncharacterized proteins from Beta vulgaris, Spinacia oleracea, Eucalyptus grandis, and Prunus persica and with SNARE-like superfamily proteins from Zostera marina and Arabidopsis thaliana. Bioinformatics analysis predicted a clathrin adaptor complex small-chain domain and $\mathrm{N}$-myristoylation site in the SbSLSP protein. Subcellular localization studies indicated that the SbSLSP protein is mainly localized in the plasma membrane. Using transgenic tobacco lines, we establish that overexpression of SbSLSP resulted in elevated tolerance to salt and drought stress. The improved tolerance was confirmed by alterations in a range of physiological parameters, including high germination and survival rate, higher leaf chlorophyll contents, and reduced accumulation of $\mathrm{Na}^{+}$ion and reactive oxygen species (ROS). Furthermore, overexpressing lines also showed lower water loss, higher cell membrane stability, and increased accumulation of proline and ROS-scavenging enzymes. Overexpression of SbSLSP also enhanced the transcript levels of ROS-scavenging and signaling enzyme genes. This study is the first investigation of the function of the SbSLSP gene as a novel determinant of salinity/drought tolerance. The results suggest that SbSLSP could be a potential candidate to increase salinity and drought tolerance in crop plants for sustainable agriculture in semi-arid saline soil.

Keywords: abiotic stress, SNARE-like superfamily protein, clathrin adaptor protein complex, clathrin-coated vesicles, halophyte, Salicornia brachiata, salt-inducible gene 


\section{INTRODUCTION}

The human population is growing rapidly and expected to be more than 9 billion by 2050 (Godfray et al., 2010). In the mission to meet food demand for the ever-increasing world population, adverse environmental factors are becoming a major challenge for the scientific community. The environmental factors affecting crop yield are mainly grouped as biotic and abiotic stresses. Salinity, drought, high temperature, waterlogging, high light intensity and the mineral deficiency are the major abiotic stresses which negatively affects plant growth, resulting in reduced yield or may leads to death of the plants at extreme conditions (Mahajan and Tuteja, 2005). These stresses are responsible for reducing yield by more than $50 \%$ of major crops across the globe (George et al., 2012). Plants respond to environmental cues via activation of various defense mechanisms that include environmental stress signal perception, transduction pathways and, upregulation/downregulation of numerous genes, and synthesis of protective molecules (Zhu, 2002; Huang et al., 2011). The roles of many stress responsive genes and micro RNAs and few of the cis- and trans- regulatory factors have been studied in stress tolerance (Yamaguchi-Shinozaki and Shinozaki, 2006; Agarwal and Jha, 2010; Nakashima et al., 2012; Singh and Jha, 2014).

Most of the earlier studies to understand the salinity tolerance mechanism have been focused on glycophytes, except some of the recent studies that include halophytes (Agarwal et al., 2013). The halophytes have unique genetic makeup (Agarwal et al., 2010), which accounts for its better tolerance mechanisms to thrive in saline environment than the glycophytes (Gong et al., 2005) and thus emerged as model organism to study the salinity tolerance mechanism in plants.

The expressed sequence tag (EST) database served as potential resource to identify novel gene(s) contributing to specific biological processes (Jha et al., 2009; Yadav et al., 2012a). The EST database of different plant species showed a major portion of unknown/ hypothetical genes that lack any similarity with known genes in the NCBI database (Yadav et al., 2014). The unknown/hypothetical novel genes identified from the EST databases can be chosen for crop genetic engineering for enhanced stress tolerance. Recently, several genes from different halophytes have been reported to increase the salinity as well as drought stress tolerance in crop, tobacco or Arabidopsis (Himabindu et al., 2016 and references therein). For example, Li et al. (2011) has found that the transgenic alfalfa plants overexpressing Salsola soda NHX1 (SsNHX1) has better salinity tolerance and survive well for 50 days in the soil irrigated with $400 \mathrm{mM}$ saline solution twice a week. Transgenic hybrid poplar plants overexpressing Populus trichocarpa PtSOS2 showed increased plasma membrane $\mathrm{Na}^{+}$exclusion activity and better ROS scavenging compared to WT plants (Yang et al., 2015). Not only the transporter genes, overexpression of a halophytic ASR1 gene and NAC transcription factor into Arabidopsis thaliana which are cloned from Suaeda liaotungensis had improved the salinity, drought, and low-temperature tolerance (Hu et al., 2014; $\mathrm{Li}$ et al., 2014).
Salicornia brachiata Roxb., an extreme annual halophyte grows in saline $(0.1-2.0 \mathrm{M})$ coastal land and can store salt as high as $40 \%$ of its dry weight (Tiwari et al., 2015). These unique characteristics of this plant may enable it as a potential model plant to study salt-responsive genes. Earlier, we characterized some of the genes including SbMAPKK (Agarwal et al., 2010), SbDREB2A (Gupta et al., 2010), SbGST (Jha B. et al., 2011), SbNHX1 (Jha A. et al., 2011), SbASR1 (Jha et al., 2012), SbMT-2 (Chaturvedi et al., 2012), SbSI-1 (Yadav et al., 2012a), SbSOS1 (Yadav et al., 2012b), SbUSP (Udawat et al., 2013, 2016), and SbSI-2 (Yadav et al., 2014) from the EST database of S. brachiata (Jha et al., 2009) and confirmed their role in abiotic stress tolerance. Therefore, these genes may serve as potential candidate for bioengineering of other crops to enhance their abiotic stress tolerance. In light of the above facts, here we have cloned and characterized another salt-inducible gene SbSLSP and its promoter from S. brachiata. Bioinformatics analysis predicted abiotic stress related cis-regulatory motifs in promoter region, which is further confirmed with higher transcript expression of SbSLSP in salt and drought stress. Subcellular localization studies indicated that the SbSLSP protein is mainly localized in the plasma membrane. Further we confirmed SbSLSP role in stress tolerance through a transgenic approach in tobacco plants. The enhanced tolerance of transgenic tobacco was established by various morphological, physiological and biochemical parameters. Overexpression of SbSLSP also enhanced the transcript levels of ROS-scavenging and signaling enzyme genes. This study provides novel insights into the function of SbSLSP gene in response to salinity and drought stress.

\section{MATERIALS AND METHODS}

\section{Plant Growth and Stress Treatments}

The Salicornia brachiata Roxb. seeds were harvested from dried plants collected from the coastal area near Bhavnagar (latitude $21^{\circ} 45^{\prime} \mathrm{N}$, longitude $72^{\circ} 14^{\prime} \mathrm{E}$ ), Gujarat, India. The seeds were germinated in plastic pots containing garden soil, and the plants were grown in natural conditions. One-month-old seedlings were carefully uprooted and transferred to hydroponic culture $1 / 2$ strength Murashige and Skoog (MS) salts (Murashige and Skoog, 1962) in a culture room with a dark/light cycle of $8 / 16 \mathrm{~h}$ at $25^{\circ} \mathrm{C}$ for 1 month. The nutrient solution was renewed twice a week. Plants were given stress treatment using $250 \mathrm{mM} \mathrm{NaCl}$ in $1 / 2$ MS solution, and desiccated by wrapping the root in tissue paper for $0,6,12$, and $24 \mathrm{~h}$. Upon completion of the treatments, shoot tissues were collected, frozen in liquid nitrogen and stored at $-80^{\circ} \mathrm{C}$.

\section{Cloning of the SbSLSP Gene and Its Putative Promoter}

The EST of SbSLSP was made full length and characterized for its role in abiotic stress tolerance. The total RNA was extracted from shoot tissues of the salt-stressed plants of S. brachiata by the GITC method (Chomczynski and Sacchi, 1987). The 5'-RACE reaction and PCR amplification was performed using primers GSPR1, 
GSPR2, GSPR3, AAP, and AUAP according to the manufacturer's protocol (Invitrogen, USA). The amplicon was purified from agarose gel and cloned into the pGEM-T Easy vector system II (Promega, Madison, Wisconsin) and sequenced (Macrogen Inc., Seoul, South Korea). After determining the open reading frame (ORF), the full-length SbSLSP cDNA was PCR-amplified with AccuPrime Pfx DNA polymerase (Invitrogen, USA) in conjunction with primers SbSLSPF and SbSLSPR containing HindIII and XhoI sites, respectively. The amplification product was then cloned into a pJET1.2/blunt cloning vector (MBI Fermentas) and sequenced. The gene sequence was submitted to the NCBI database (accession number KF111691).

Three gene-specific primers named SbSLSPPR1, SbSLSPPR2, and SbSLSPPR3 were designed from the cDNA sequence. Then, the putative promoter region was isolated using the genomewalking technique (Tiwari et al., 2014, 2016). The putative promoter was sequenced and submitted to NCBI (accession number KP229524). See Table S1 for primer sequences.

\section{In silico Analysis}

The NCBI database was used as a search engine for nucleotide and protein sequences. TMpred online software was used for the prediction of transmembrane domains and ClustalW software was used for sequence alignment. Conserved domains of SbSLSP were determined by BLASTp programme (http://www.ncbi.nlm.nih.gov). Secondary structure prediction of SbSLSP was carried out by Expasy tools (http://www.expasy.ch/tools/), while the phosphorylation motifs were predicted by NetPhosK 1.0 server. The Pfam and SMART programs were used for conserved domain analysis. The relationship of SbSLSP with its homologs from other plant species was inferred by constructing a phylogenetic tree using MEGA Ver. 6.1. The robustness in topology of the phylogenetic tree was assessed based on bootstrap values. In silico analysis of the putative promoter fragment was performed for the presence of cis-regulatory elements using the online programs PLACE (http://www.dna.affrc.go.jp/PLACE) and PlantCARE (http://bioinformatics.psb.ugent.be/webtools/plantcare/html/).

\section{Quantitative Real-Time PCR (qRT-PCR) Analysis}

Total RNA was isolated from S. brachiata control and treated plant samples using the GITC method (Chomczynski and Sacchi, 1987) and qRT-PCR of SbSLSP was performed as previously described Yadav et al. (2014) using primer pair SbSLSP_RTF1 and SbSLSP_RTR1. The $\beta$-tubulin gene was used as an internal control and ampliflied using primers BTF and BTR. Samples were amplified in triplicate and independent experiments were repeated three times. Fold changes were calculated using the $2^{-\Delta \Delta \mathrm{C}_{\mathrm{T}}}$ method (Livak and Schmittgen, 2001). See Table S1 for primer sequences.

\section{Subcellular Localization of SbSLSP Protein}

The localization fusion construct with RFP (red fluorescent protein) was made using Gateway technology. The full-length SbSLSP cDNA was PCR-amplified with AccuPrime Pfx DNA polymerase in conjunction with SbSLSPCAF and SbSLSPCAR primers. The blunt-ended PCR product was then cloned into a $P E N T E R / D-T O P O$ entry vector and then into the destination vector $p S I T E-4 C A$ by Gateway LR Clonase II enzyme mix (Yadav et al., 2014; Invitrogen, USA). The fusion construct (RFP:SbSLSP) was transferred into onion epidermal cells by particle bombardment (PDS-1000/He Biolistic, Biorad, USA). The pSITE-4CA (RFP) vector was used as a control. After incubation on an MS plate for $12-24 \mathrm{~h}$, the onion epidermal cells were observed for transient expression of RFP with an epifluorescence microscope (Axio Imager, Carl Zeiss AG, Germany). See Table S1 for primer sequences.

\section{Construction of Plant Transformation Vector and Tobacco Transformation}

To perform plant transformation, SbSLSP cDNA was PCRamplified with AccuPrime Pfx DNA polymerase in conjunction with SbSLSPPF and SbSLSPPR primers that contained ApaI and $K p n I$ sites, respectively. The SbSLSP gene was cloned into the pRT100 vector (Topfer et al., 1987) to attach the $35 S$ promoter and terminator. The above gene expression cassette (35S-SbSLSPterminator) was sub-cloned into the pCAMBIA2301 binary vector at the PstI site. The resultant construct $P C A M B I A 2301-$ 35S:SbSLSP and PCAMBIA2301 alone were mobilized into Agrobacterium tumefaciens (LBA 4404) for tobacco (Nicotiana tabacum var. Xanthi) transformation (Horsch et al., 1985). Independently regenerated and kanamycin resistant $(50 \mathrm{mg} / \mathrm{l})$ transgenic lines $\left(\mathrm{T}_{0}\right)$ were propagated and screened using GUS assay ( $\beta$-Glucuronidase Reporter Gene Staining Kit; SigmaAldrich) and PCR analysis (Yadav et al., 2014). By selection with resistance to kanamycin $(100 \mathrm{mg} / \mathrm{l})$ at the seedling stage of $\mathrm{T}_{1}$ and $\mathrm{T}_{2}$ plants, were harvested for subsequent experiments. At $\mathrm{T}_{1}$ generation, lines which showed 3:1 ratio of resistant to sensitive on the MS plates supplemented with kanamycin $(100 \mathrm{mg} / \mathrm{L})$ were selected and grown to harvest the seed of next generation. At $\mathrm{T}_{2}$ generation, the lines which seeds shown $100 \%$ resistance on antibiotic plates was chosen for the further analysis. See Table S1 for primer sequences.

\section{Molecular Analysis of Transgenic Lines}

The presence of the transgene was confirmed by PCR using a SbSLSP-specific primer pairs SbSLSP_RTF2 and SbSLSP_RTR2. To confirm the expression of GUS activity, histochemical staining was conducted as described previously (Yadav et al., 2014). To check the transcript expression of the overexpressed SbSLSP gene in transgenic plants, reverse-transcriptase PCR (RT-PCR) was carried out. Total RNA was isolated from wild-type (WT) and transgenic plant samples, and cDNA was prepared. The RT-PCR was performed as previously described (Yadav et al., 2014) using the SbSLSP-specific primer pair. The primer pair QACTF and QACTR was used for actin as the internal control. See Table S1 for primer sequences.

\section{Evaluation of Transgenic Plants Exposed to Salt and Osmotic Stress}

To analyse the stress tolerance of SbSLSP-overexpressing tobacco plants $\left(T_{2}\right)$, the seeds were germinated on MS agar medium supplemented with $200 \mathrm{mM} \mathrm{NaCl}$ (salt stress) or $300 \mathrm{mM}$ 
mannitol (osmotic stress) in culture room conditions. The percentage of seed germination was scored for 9 days at 2-day intervals. For study of seedling root morphology, the germinated seeds of transgenic and WT plants were grown on vertical plates for 10 days in MS media and 21 days for MS media supplemented with $200 \mathrm{mM} \mathrm{NaCl}$ or $300 \mathrm{mM}$ mannitol. The primary root lengths and fresh weights were recorded, and physiological changes were observed at the end of the culture periods. For salinity and drought tolerance assays in plant, $\mathrm{T}_{2}$ transgenic lines and WT seeds were germinated on MS medium. Ten-day-old seedlings were planted in identical pots containing soilrite mix (horticulture-grade expanded perlite, Irish Peat moss and exfoliated vermiculite in equal ratio) and were well watered with 1/8 MS salt solution for 20 more days in the growth chamber $\left(22^{\circ} \mathrm{C}, 60 \%\right.$ humidity, $16 \mathrm{~h}$ light $/ 8 \mathrm{~h}$ dark cycle $)$. The transgenic and WT plants with similar size and growth were divided into four groups. Plants were watered every 2 days with 1/8 MS salt solution supplemented with (three groups) or without (one group) $250 \mathrm{mM} \mathrm{NaCl}$ for 18 days. In another set of drought stress assays, plants irrigation was stopped for 18 days and recovered after re-watering for next 5 days. During these treatments, morphological changes were observed and photographed, and the survival rate was measured. The leaves, stems and roots of each plant were harvested separately for $\mathrm{Na}^{+}$and $\mathrm{K}^{+}$determination. Leaf-disc assays to determine the total chlorophyll content of transgenic and WT plants were performed with protocols as described in earlier reports (Porra et al., 1989; Chaturvedi et al., 2014). In another experiment, 6week-old plants were subjected to $1 / 4 \mathrm{MS}$ salt solution containing $\mathrm{NaCl}(250 \mathrm{mM})$ or PEG-6000 (20\%, w/v) stress in hydroponic conditions for 3 weeks. MS salt solution containing $\mathrm{NaCl}$ and PEG were replaced every second day during the stress assay, and photographs were taken. Consistent results were observed in three independent experiments. The histochemical detection of $\mathrm{H}_{2} \mathrm{O}_{2}$ in the leaves of NaCl- or PEG-treated plants for 12 $\mathrm{h}$ was measured as described by Mukherjee and Choudhuri (1983). All experiments were repeated with three experimental and biological replicates. Each biological replicate contained 10 plants.

\section{Determination of $\mathrm{Na}^{+}$and $\mathrm{K}^{+}$Content}

Plant materials collected at the end of salt treatment were used for determination of $\mathrm{Na}^{+}$and $\mathrm{K}^{+}$content via the method previously described by Shukla et al. (2012).

\section{Measurement of Various Biochemical and Physiological Parameters}

Three-week-old seedlings were subjected to salinity $(250 \mathrm{mM}$ $\mathrm{NaCl}$ ) or osmotic (20\% (w/v) PEG-6000) stress for 1, 6, 12, and 24 h. Cell membrane stability (CMS), relative water content (RWC), proline, and $\mathrm{H}_{2} \mathrm{O}_{2}$ content were measured from fresh samples after stress recovery (Bates et al., 1973; Wang et al., 2011; Garg et al., 2012). MDA and activity of antioxidant enzymes (APX: EC 1.11.1.11 and GR: EC 1.6.4.2) were performed with stressed samples stored at $-80^{\circ} \mathrm{C}$ (Garg et al., 2012). Ten seedlings were pooled as one sample, and three replicates of samples were taken for all lines in each experiment.

\section{Expression Analysis of Reactive Oxygen Species (ROS)-Scavenging and Signaling Genes}

The expression patterns of ROS-scavenging (NtSOD: EC 1.15.1.1, NtAPX: EC 1.11.1.11, NtPOX: EC 1.11.1.7) and signaling (NtPLC1) genes in transgenic and WT plants were also analyzed by qRT-PCR as described previously (Yadav et al., 2014). The gene-specific primer pairs of NtSOD, NtAPX, NtPOX (primer sequences taken from Huang et al., 2011) and phosphoinositidespecific phospholipase C1 NtPLC1 (NtPLCF and NtPLCR primers) were utilized for the expression study. The actin gene was used as an internal control and amplified using primers QACTF and QACTR to normalize all data. Samples were amplified in triplicate, and independent experiments were repeated three times. Fold changes were calculated using the $2^{-\Delta \Delta \mathrm{C}_{\mathrm{T}}}$ method (Livak and Schmittgen, 2001). See Table S1 for primer sequences.

\section{Statistical Analyses}

One-way ANOVA using between subject factors algorithm was performed by ezANOVA (http://www.cabiatl.com/mricro/ ezanova/) programme for analysis of variance to determine the least-significant difference among means. Mean values that were significantly different within treatment group were evaluated by Duncan's test at ${ }^{*} P<0.05,{ }^{* *} P<0.01$ and ${ }^{* * *} P<0.001$.

\section{RESULTS}

\section{Isolation and Sequence Analysis of SbSLSP and Its Promoter}

The EST of SbSLSP (GenBank accession number EB484673) was made full length using $5^{\prime}$-RACE. The cDNA of SbSLSP (NCBI accession id KF111691) was 840-bp long, including a $5^{\prime}$ UTR of 60-bp, an ORF of 444-bp and a 3' UTR region of 336-bp (Figure S1). The encoded protein of SbSLSP contains 147 amino acids with an estimated molecular weight of $16.84 \mathrm{kDa}$ and a pI of 5.44 . The NCBI protein blast (blastp) analysis revealed that the SbSLSP protein has a high sequence identity (78-95\%) to a SNARElike superfamily protein and/or hypothetical uncharacterized proteins from different plant species. It has 78 and $84 \%$ sequence identity with $A$. thaliana and Zostera marina SNARE-like proteins, respectively. The SbSLSP also showed high identity with uncharacterized/ hypothetical proteins of Beta vulgaris (95\%), Spinacia oleracea (93\%), Eucalyptus grandis (85\%), and Prunus persica (84\%). Phylogenetic analysis revealed that SbSLSP showed close neighborhood with other Amaranthaceae family members B. vulgaris and S. oleracea, which is suggesting the evolutionary conservation within the family. The SbSLSP also showed close proximity with other halophyte $Z$. marina (Figure S2). The bioinformatics analysis using the Pfam program predicted a clathrin adaptor complex small-chain domain in the SbSLSP protein. The SbSLSP protein may be phosphorylated by CKII (caseine kinase II) and PKC (protein kinase C). The SbSLSP also contained an N-myristoylation site at the 125-130 amino acid position (GVleNT) (Figure S1). There was no transmembrane domain predicted in SbSLSP by hydropathicity analysis, whereas 
the SbSLSP secondary structure analysis predicted four, six and eleven alpha helixes, extended strands and random coils, respectively (Figure S3). The 872-bp putative promoter region upstream of translation start site ATG had a number of cisregulatory motifs that were categorized into six different groups: abiotic stress inducible, conserved motifs, light responsive, miscellaneous, phytohormone inducible and tissue/organellespecific expression (Table S2). Sequence analysis revealed the presence of several cis-regulatory motifs related to abiotic stress responses, like anaerobic, drought, metal, salinity, and high temperature stresses, in the promoter region (Table S3; Figure S4).

\section{Differential Expression of SbSLSP Transcripts under Salinity and Desiccation Stress}

The expression profile of the SbSLSP gene was carried out by qRT-PCR in S. brachiata. In the presence of $250 \mathrm{mM} \mathrm{NaCl}$, the transcript of SbSLSP increased by 6 -fold at $6 \mathrm{~h}$ and it remain unchanged at $12 \mathrm{~h}$ and then increased up to 16 -fold at $24 \mathrm{~h}$ time-point of stress treatment (Figure 1A). In desiccation stress, the transcript was up-regulated from 10 - to 25 -fold and the maximum expression was observed at $24 \mathrm{~h}$ time-point (Figure 1B).

\section{The SbSLSP Protein is Localized to the Plasma Membrane}

The transient expression assay into onion epidermal cells was performed to determine the in vivo subcellular localization of SbSLSP using RFP alone or the RFP:SbSLSP fusion construct into $p S I T E-4 C A$ vector (Figure 1C). Onion cells were transformed with $R F P$ alone, displayed even distribution of red fluorescence signals in the entire cell region, whereas in RFP:SbSLSP transformed cells, the fluorescence was predominantly accumulated in the plasma membrane and only slightly in the cytoplasmic region (Figure 1D). These results clearly indicate that SbSLSP protein is mostly localized in the plasma membrane and to some extent in the cytoplasmic region.

\section{Analysis of SbSLSP-Overexpressing Transgenic Tobacco Plants}

The tobacco plants were genetically transformed with pCAMBIA2301-35S:SbSLSP construct (Figure 1C) and pCAMBIA2301 empty vector for in vivo functional characterization of the SbSLSP. Total 35 independent transgenic lines which were resistant to kanamycin were also confirmed by PCR using gene-specific as well as gus-specific primers (data not shown). The empty vector-transformed transgenic (VC) and WT plants were used as controls. The germination assay of seeds from these $T_{1}$ transgenic plants on kanamycin-supplemented medium exhibited the ratio of 3:1 $\left(\operatorname{Kan}^{\mathrm{r}} / \operatorname{Kan}^{\mathrm{s}}\right)$. The $\mathrm{T}_{2}$ generation lines were again verified by PCR amplification. At $\mathrm{T}_{2}$ generation, the three 35S:SbSLSP GUS-positive and one empty-vector transgenic lines (L3, L28, L29, VC) were chosen for subsequent analysis (Figure 1E). The SbSLSP-overexpressing transgenic lines exhibited different levels of SbSLSP expression via RT-PCR, whereas there were no expression of SbSLSP was detected in WT and VC plants (Figure 1F). The transgene transcript expression detected in L28 transgenic line was maximum among other lines used in the analysis (Figure 1F).

\section{Salinity and Osmotic Stress Responses of SbSLSP-Overexpressing Transgenic Lines}

The effect of salinity and osmotic stress on germination was studied by germinating transgenic lines and WT seeds on MS medium comprising $200 \mathrm{mM} \mathrm{NaCl}$ or $300 \mathrm{mM}$ mannitol. In the medium containing $\mathrm{NaCl}$, the maximum germination rate for WT and VC were 40 and 39\%, respectively, while for overexpressing lines, more than $89 \%$ germination was recorded (Figure 2A). In the medium containing mannitol, the maximum germination rate for WT and VC was 46.8 and $37.5 \%$, respectively, while the germination rate was more than $92 \%$ for transgenic lines (Figure 2B). In addition to seed germination assays, physiological changes were observed. The seedlings of WT, VC and overexpressing lines were shifted to MS medium with or without $200 \mathrm{mM}$ salt or $300 \mathrm{mM}$ mannitol. We measured the primary root length after 10 days, and observed that primary roots were almost equal in all the lines growing at $1 / 2$ MS (Figures 2C,D). The physiological changes of the $\mathrm{T}_{2}$ transgenic seedlings, compared to the WT seedlings, were observed after 21 days of salinity and osmotic stresses, and SbSLSP-overexpressing lines showed significantly better growth performance (Figure 2C). After 21 days, most of the WT and VC plant growth had stopped, while the SbSLSP-overexpressing lines showed significantly higher root and shoot growth (Figures 2C,E). Furthermore, the fresh weight analysis showed that salinity and osmotic stress had low adverse effect on the SbSLSPoverexpressing lines, while significant growth impairment was seen in the biomass synthesis of WT and VC plants (Figure 2F).

\section{Analysis of SbSLSP-Overexpressing Plants $\left(T_{2}\right)$ in Salt and Drought Stress}

To characterize the performance of $S b S L S P$-overexpressing plants under salinity and drought stress, 10-day-old seedlings were grown in Soilrite for an additional 20 days. Thereafter, plants were treated with $250 \mathrm{mM} \mathrm{NaCl}$ for 18 days to induce salt stress and irrigation was stopped for 18 days to induce drought stress. The signs of stress, such as wilting symptoms, were more evident in WT and VC than those of the SbSLSPoverexpressing plants for both the stresses (Figures $\mathbf{3 A}, \mathbf{B}$ ). In the presence of salinity stress, more than $83 \%$ of SbSLSPoverexpressing plants survived, compared to only $41 \%$ survival for WT and VC plants (Figure 3C). Five days after re-watering to drought stressed plants, $65-83 \%$ of $S b S L S P$-overexpressing plants survived, whereas the survival rate was 33\% for WT and VC plants (Figure 3C). We also performed leaf-disc assays to determine chlorophyll content under stress-induced necrosis of leaf disc. The transgenic plants retained higher chlorophyll 
A

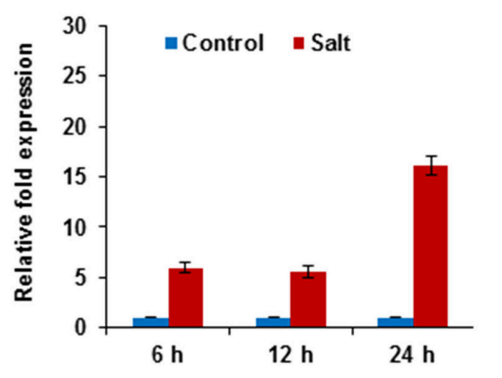

B

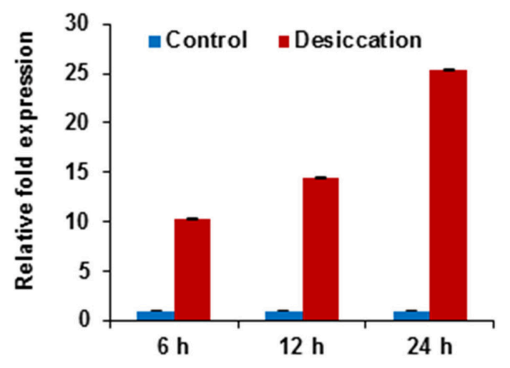

$6 \mathrm{~h}$
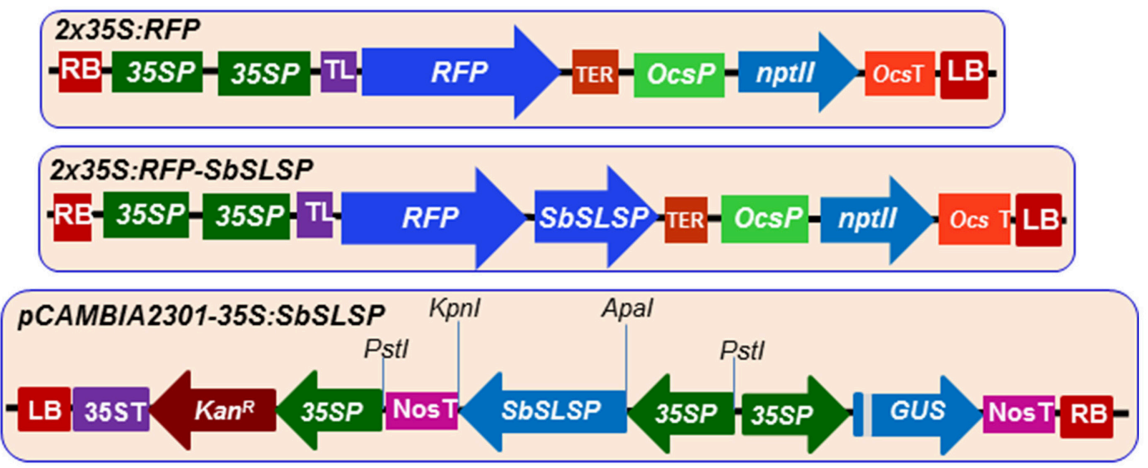

D

Bright field RF field Merged

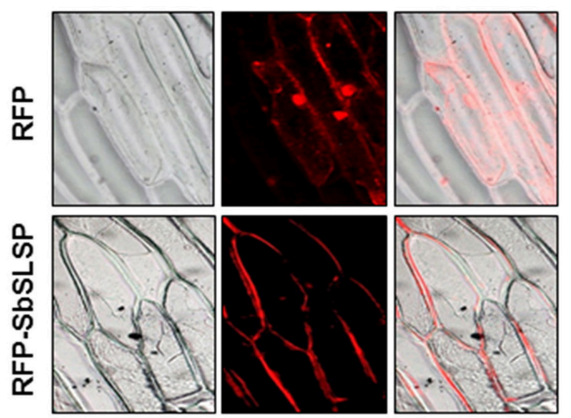

$\mathbf{F}$

E

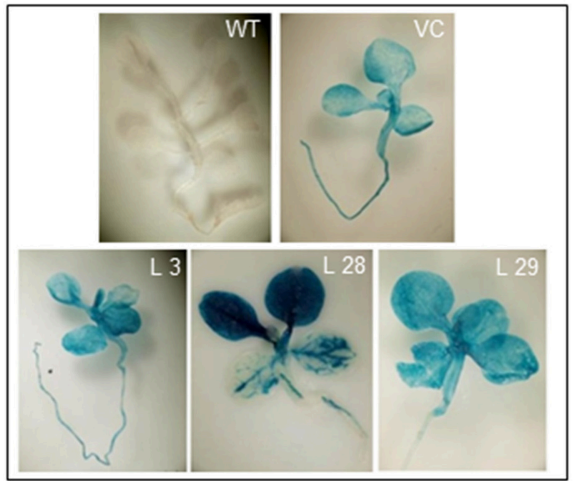

$\begin{array}{lllllll}\text { WT } & \text { VC } & \text { L3 } & \text { L7 } & \text { L9 } & \text { L28 } & \text { L29 }\end{array}$

SbSLSP

Actin

FIGURE 1 | Expression, localization and molecular analyses of SbSLSP-overexpressing transgenic tobacco plants. (A,B) Expression pattern of SbSLSP in response to salt and desiccation stress treatments for different time periods in Salicornia brachiata. The relative fold expression of SbSLSP in stressed and non-stressed conditions was calculated using the $2^{-\Delta \Delta C} \mathrm{~T}$ method. Values are means $\pm \mathrm{SD}$ from three independent measurements. (C) Schematic representation of the pSITE-3CA-2X35S:RFP:SbSLSP construct (RFP:SbSLSP) used for transient expression and pCAMBIA2301-35S:SbSLSP construct used to transform tobacco plants with the SbSLSP gene. (D) SbSLSP resides in the plasma membranes of onion epidermal cells. Cells with constructs expressing red fluorescence protein (RFP) alone and the RFP:SbSLSP fusion protein were analyzed using a bright and red fluorescence field microscope. (E) GUS assay of $T_{2}$ seedlings showing positive GUS expression in the transgenic lines. (F) Transcript levels of the SbSLSP gene in transgenic lines and WT T 2 plants via semi-quantitative RT-PCR.

contents as compared to WT and VC plants under stress treatments (Figure 3D). To evaluate the stress effects on the growth of SbSLSP-overexpressing plants, 6-week-old plants were grown in hydroponic $1 / 2 \mathrm{MS}$ salt solution supplemented with $250 \mathrm{mM} \mathrm{NaCl}$ or $20 \%$ (w/v) PEG-6000. The stressed WT and VC plants exhibited retardation of growth, whereas SbSLSPoverexpressing plants grew healthier (Figure 3E).

\section{Ion Content Analysis and $\mathrm{H}_{2} \mathrm{O}_{2}$ Accumulation of SbSLSP-Overexpressing Tobacco Plants under Stress}

To determine how SbSLSP maintains the membrane potential in transgenic tobacco plants, we studied the accumulation of $\mathrm{K}^{+}$and $\mathrm{Na}^{+}$ions in tissues of transgenic and WT plants grown under control or salt $(250 \mathrm{mM} \mathrm{NaCl})$ treated 
A

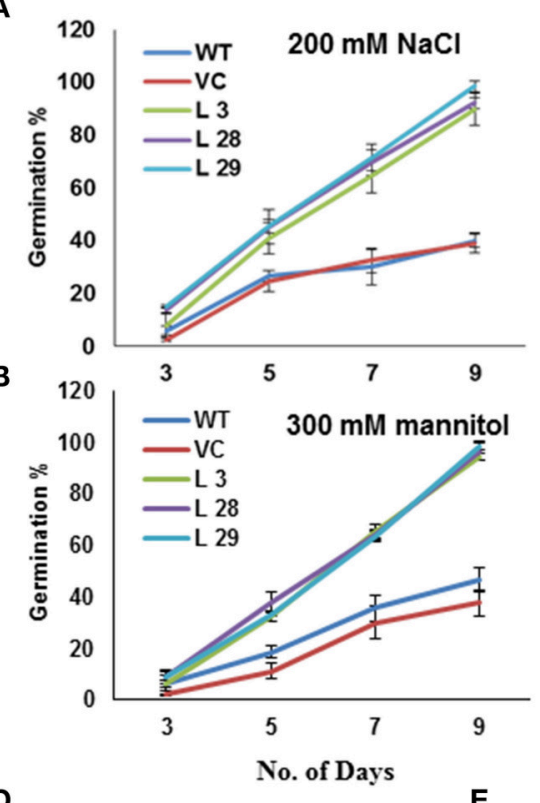

C

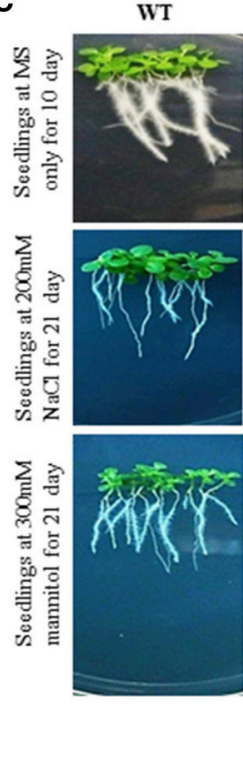

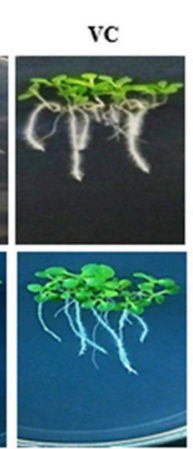

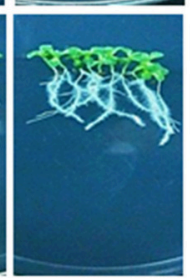

L3
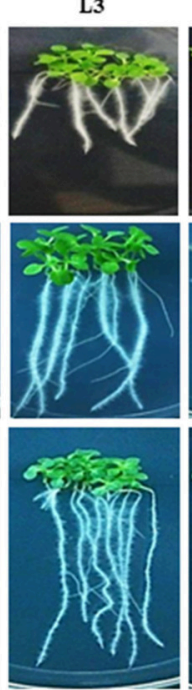
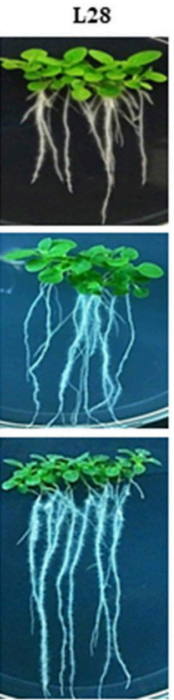

L29

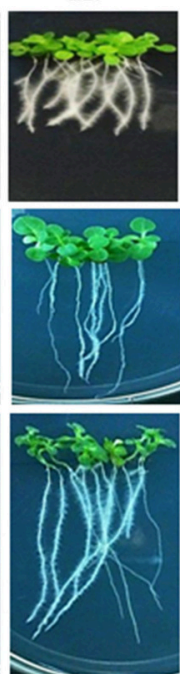

D

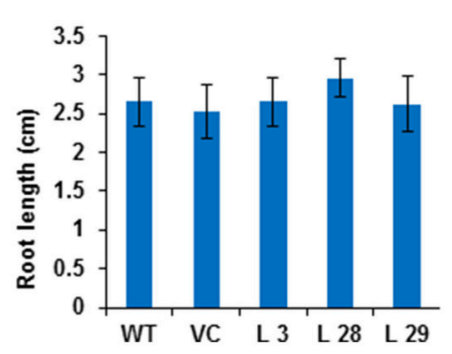

$\mathbf{E}$

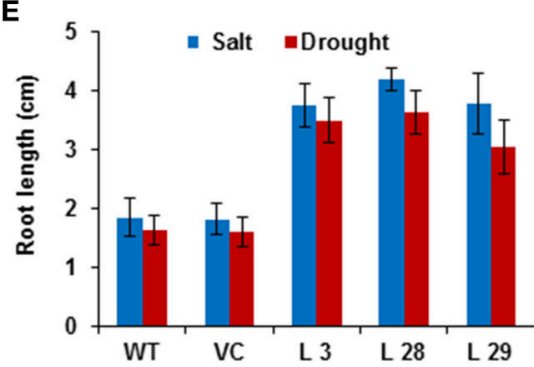

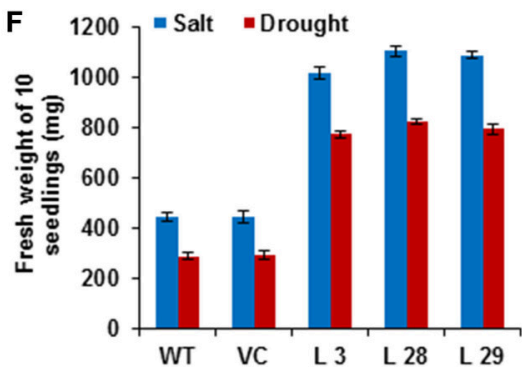

FIGURE 2 | Effect of SbSLSP on stress tolerance in $\mathbf{T}_{\mathbf{2}}$ transgenic tobacco. (A,B) Germination of SbSLSP transgenic and wild-type plants in MS medium supplemented with $200 \mathrm{mM} \mathrm{NaCl}$ or $300 \mathrm{mM}$ mannitol. The percentage of germinated seeds was calculated based on the number of seedlings that reached the cotyledon stage by 9 days. (C) Root growth comparisons of the germinated seedlings of transgenic and WT plants, which were grown on vertical plates for 10 days in MS media for control experiment and 21 days for MS media supplemented with $200 \mathrm{mM} \mathrm{NaCl}$ or 300 mM mannitol for stress experiment. (D,E) Graphical representation of root measurements of transgenic and wild-type plants in control and stress conditions (salt or drought stress), respectively. Seeds of each line were germinated and planted on MS agar, or MS agar supplemented with $200 \mathrm{mM} \mathrm{NaCl}$ or $300 \mathrm{mM}$ mannitol in triplicate. Root length was measured at 10 days for MS agar and 21 days for MS agar supplemented with $200 \mathrm{mM} \mathrm{NaCl}$ or $300 \mathrm{mM}$ mannitol. Values are the mean $\pm \mathrm{SD}$ (triplicate measurements; $n=10$ ). (F) Fresh weight measurement of 21-day-old seedlings in salt and drought stress. Values are the mean \pm SD (triplicate measurements; $n=10$ ).

conditions. The transgenic and WT plants grown under control conditions exhibited almost equal $\mathrm{Na}^{+}$content (Figure 4A). After salinity stress, the $\mathrm{Na}^{+}$ion accumulation was higher in the leaves, stem, and root tissues of the WT and transgenic plants than the plants grown under control conditions, but SbSLSP-overexpressing plants accumulated lower $\mathrm{Na}^{+}$ compared to WT and VC plants (Figure 4A). An increased $\mathrm{K}^{+}$content was observed in SbSLSP-overexpressing plants compared with WT and $\mathrm{VC}$ under $\mathrm{NaCl}$ stress (Figure 4B). The SbSLSP-overexpressing plants showed a significantly improved $\mathrm{K}^{+} / \mathrm{Na}^{+}$ratio in the leaves, stem, and root tissues relative to WT and VC (Figure 4C). As for accumulation of $\mathrm{H}_{2} \mathrm{O}_{2}$ during stress conditions, histochemical staining of leaves with diaminobenzidine showed more intense staining in WT and VC plants compared to SbSLSP-overexpressing plants (Figure 4D).

\section{Overexpression of the SbSLSP Gene Improves the ROS-Scavenging Mechanism under Salinity and Drought Stress}

The abiotic stresses exert secondary oxidative stress to plants besides the ionic toxicity, which results in peroxidation of the membrane lipids. The degree of lipid peroxidation, ROSaccumulation and integrity of cell membranes under salinity and drought stresses were determined by quantifying the accumulation of malondialdehyde (MDA) and $\mathrm{H}_{2} \mathrm{O}_{2}$, and the CMS in $\mathrm{T}_{2}$ transgenic seedlings. Simultaneously, accumulation of proline was also estimated under same conditions. Under salinity- and drought-stress conditions, there was significantly less accumulation of $\mathrm{MDA}$ and $\mathrm{H}_{2} \mathrm{O}_{2}$, and higher CMS and proline in transgenic lines compared to WT and VC plants, confirming reduced damage, higher membrane integrity and better osmotic adjustments of SbSLSP-overexpressing plants 


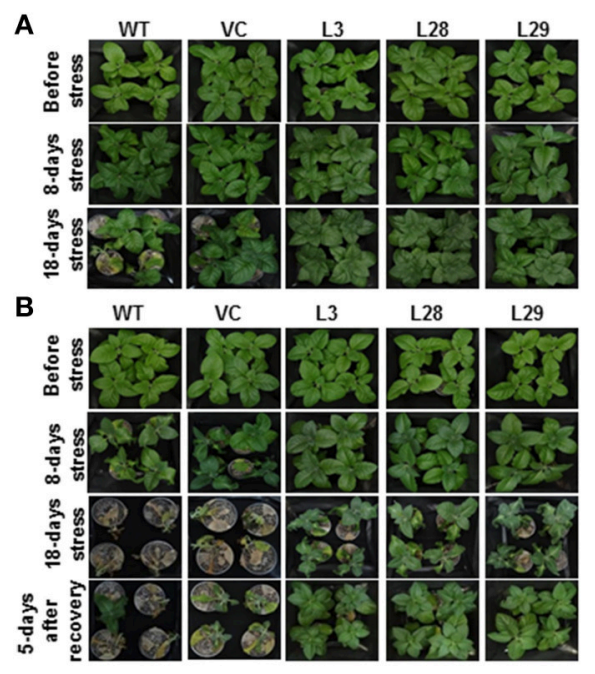

C

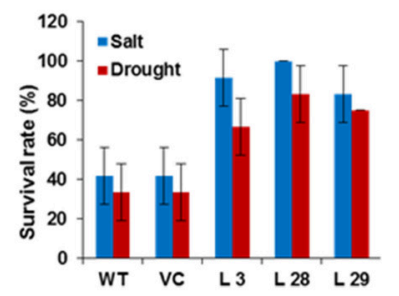

D
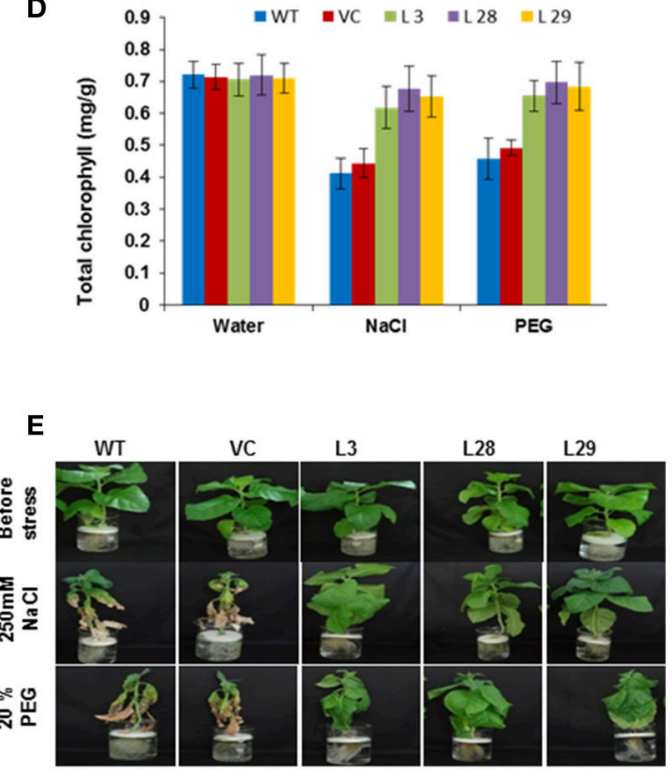

FIGURE 3 | Response of wild-type (WT), vector control (VC), and SbSLSP T2 transgenic tobacco plants under salt and drought stress. (A) Five-week-old transgenic lines $(\mathrm{L} 3, \mathrm{~L} 28, \mathrm{~L} 29, \mathrm{VC})$ and $\mathrm{WT}$ were irrigated with $\mathrm{NaCl}$ solution $(250 \mathrm{mM})$ for 18 days. Photographs of representative seedlings were taken at the initial stage of salt stress, 8 days after stress, and 18 days after stress. (B) The water supply of 5-week-old normal-growing transgenic lines (L 3, L-28, L-29, VC) and WT was depleted 18 days. Photographs of representative seedlings were taken at the initial stage of drought stress, 8 days after stress, 18 days after stress, and 5 days after re-watering. (C) Quantitative analysis of survival of transgenic lines and WT plants during stress. Values are the mean \pm SD (triplicate measurements; $n=4$ plants). (D) Quantification of chlorophyll retention from the leaf-disc assay. Values are the mean \pm SD of three independent assays. (E) Representative photographs of 6-week-old transgenic plants ( L 3, L 28, L 29, VC) grown in $250 \mathrm{mM} \mathrm{NaCl}$ or 20\% PEG compared to WT for 3 weeks.

(Figures 5A-D, 6A-D, respectively). To confirm improved ROSscavenging mechanisms under stress conditions, the activity of oxidative enzymes was also analyzed. The activity of glutathione reductase (GR, EC 1.6.4.2) and ascorbate peroxidase (APX, EC 1.11.1.11) increased markedly in SbSLSP-overexpressing seedlings compared to that of WT and VC plants (Figures 5E,F, 6E,F). Furthermore, a higher relative water content was found in SbSLSP-overexpressing seedlings compared to WT and VC seedlings (Figures 5G, 6G). These data indicate improved capacity of osmotic adjustment in transgenic tobacco seedlings.

\section{Expression of ROS-Scavenging and Signaling Genes in SbSLSP-Overexpressing Tobacco Plants}

To further elucidate the mechanism(s) behind the improved stress tolerance of $S b S L S P$-overexpressing plants, we carried out expression analysis of stress-responsive genes. The transcript levels of ROS-scavenging genes NtSOD, NtAPX, NtPOX, and phosphatidic acid mediated signaling pathway enzyme gene NtPLC1 were higher in SbSLSP-overexpressing plants than that of WT and VC plants under both salinity and drought stresses
(Figures 7A-D). Under control conditions, the transgenic lines also showed higher expression of ROS-scavenging and NtPLC1 genes. These results suggest that the SbSLSP overexpression regulates the expression of these genes.

\section{DISCUSSION}

High salinity and drought impede crop growth, survival and productivity. Plants adapted to survive stresses via mechanisms that operate at the whole plant, tissue, organ, cellular, and molecular level. Plant's plasticity to salt stress comprises a number of genes encoding proteins which involved in ion homeostasis, osmolyte synthesis, and antioxidant synthesizing enzymes (Agarwal et al., 2013). Being more efficient to survive saline conditions than glycophyte, halophytic plants become a potential bioresource for novel salt-responsive genes. Large numbers of unknown or hypothetical genes are reported in the EST database of many halophytic plants (Yadav et al., 2014). Among the ESTs database of S. brachiata (Jha et al., 2009), SbSLSP showed higher up-regulation upon salinity stress and desiccation (Figures 1A,B), which was supported by the in silico analysis 

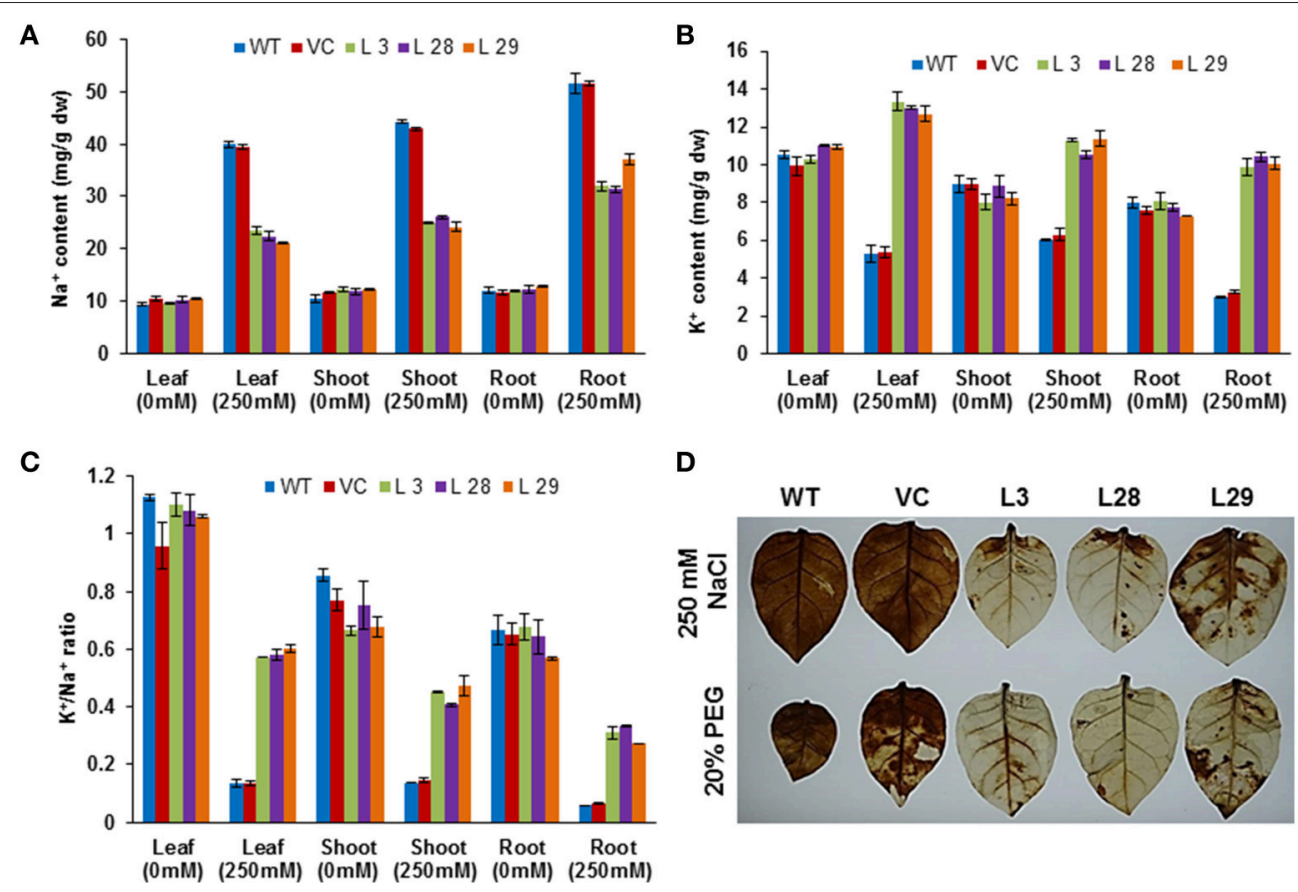

FIGURE 4 | lon content and detection of $\mathrm{H}_{2} \mathrm{O}_{2}$ in $\mathrm{T}_{2}$ transgenic and wild-type tobacco plants. (A) $\mathrm{Na}^{+}$content in leaves, shoots and roots of transgenic and wild-type plants. (B) $\mathrm{K}^{+}$content in leaves, shoots and roots of transgenic and wild-type plants. (C) $\mathrm{K}^{+} / \mathrm{Na}^{+}$ratio with or without salt treatment. Values are means \pm SD from three independent trials. (D) In vivo localization of stress-induced $\mathrm{H}_{2} \mathrm{O}_{2}$ production in leaves of WT, VC and SbSLSP-overexpressing transgenic lines after stress treatment by staining with diaminobenzidine.

of the putative promoter, containing stress-related cis-regulatory elements (Table S2). The expression of SbSLSP is controlled via an ABA-mediated signaling pathway, which includes MYBdependent induction. Besides MYB- and MYC-binding elements, the promoter region showed other stress-responsive elements, including salinity, cold, and heat shock-responsive CAAT boxes (Table S3; Figure S4). It was interesting that a large number of motifs were detected that are responsible for root nodule-specific expression (Table S2). This suggests that, apart from the stressresponsive expression, SbSLSP may also play a crucial role in nutrient absorption and nitrogen assimilation.

The NCBI protein blast analysis revealed that the SbSLSP protein has a high sequence identity to a SNARE-like superfamily protein from Z. marina and A. thaliana. Phylogenetic analysis also revealed that the SbSLSP has close proximity with halophyte Z. marina (Figure S2). More closeness of SbSLSP protein with $Z$. marina homolog as compared to Arabidopsis suggests a common plasticity mechanism in halophytes. The SbSLSP also showed high sequence identity (80-95\%) with hypothetical/uncharacterized proteins from different plant species. But protein blastp analysis against the Arabidopsis proteins displayed only similarity with the SNARE-like protein. SNAREs are fundamental controllers of membrane trafficking machinery and are playing crucial role in many biological processes such as growth, ion homeostasis, hormone and stress signaling (Lipka et al., 2007; El Kasmi et al., 2013). The SNARE proteins also play vital role to cope-up with the abiotic stresses
(Zhu et al., 2002; Mazel et al., 2004; Leshem et al., 2006; Hamaji et al., 2009; Kim and Bassham, 2011; Bao et al., 2012; Uemura et al., 2012; Tarte et al., 2015).

In silico analysis predicted an N-myristoylation site at 125130 amino acid position (GVleNT) in the SbSLSP protein (Figure S1). Myristoylation plays a pivotal role in membrane targeting by anchoring proteins to the membrane, and in abiotic stress signaling in plant responses to environmental stresses. The SbSLSP protein also has a conserved domain for clathrin AP complex small-chain domain. The AP complexes are cytosolic heterotetramers which involve in the assembly of clathrin-coated vesicles (CCVs). The CCVs play crucial role in transportation of membrane proteins via endocytic and secretory pathways (Nakatsu and Ohno, 2003). In the subcellular localization study, the SbSLSP protein mainly localized to the plasma membrane (Figure 1D), which supporting our in-silico analysis result. The SbSLSP showed similarity with SNARE-like proteins and has AP complexe small-chain domain. Plants subjected to osmoticstress, experience alterations in cell membrane dynamics and lipid arrangement to uphold cellular integrity and to reduce ion leakage (Koning et al., 2008). In the present study, the $S b S L S P$-overexpressing transgenic plants experiencing salinity and osmotic stress exhibited higher cell membrane stability hence less leakage. The bulk-flow endocytosis prevents the excessive osmotic water loss by internalization of the additional surface area and also helps to regain the turgor pressure (Staehelin and Newcomb, 2000). The SbSLSP-overexpressing transgenic lines 

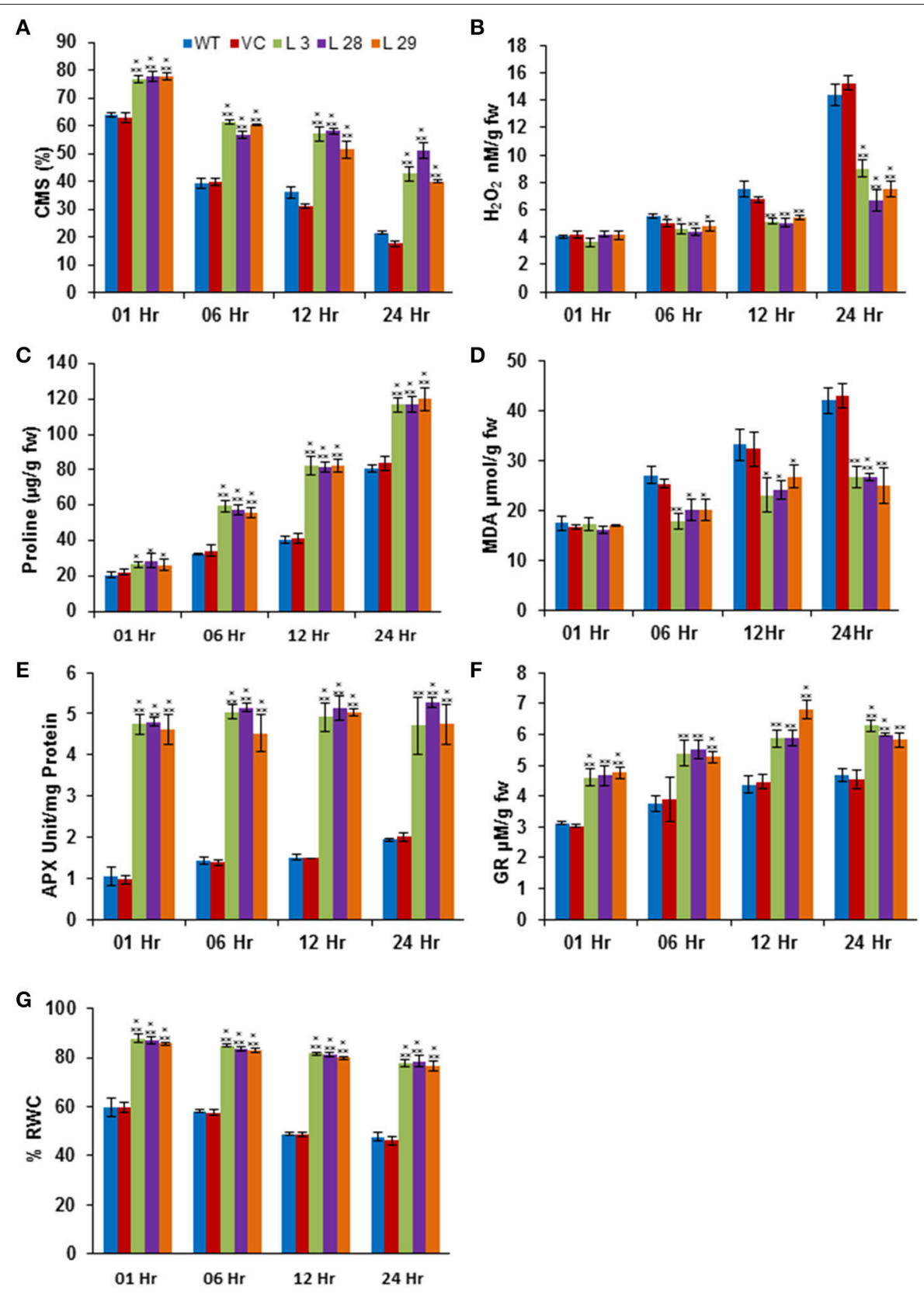

FIGURE 5 | Biochemical and physiological responses of SbSLSP transgenic plants, and VC and WT under salinity stress (250 mM NaCI). (A) Percentage of cell membrane stability. (B) Changes in hydrogen peroxide content. (C) Changes in the level of proline accumulation. (D) Levels of lipid peroxidation expressed in terms of MDA content. (E) Changes in ascorbate peroxidase enzyme activity. (F) Changes in glutathione reductase enzyme activity. (G) Percentage of relative water content. One-way analysis of variance (ANOVA) was used to test the significance between the mean values of control and transgenic plants, and comparison among mean values was performed using ezANOVA (http://www.cabiatl.com/mricro/ezanova/). The difference between control and transgenic lines were statistically significant at ${ }^{*} P<0.05,{ }^{* *} P<0.01$ and ${ }^{* \star *} P<0.001$.

showed better water status relative to the WT plants (Figures 5G, 6G). Phosphatidic acid (PA) mediated signaling pathway plays vital role in plant stress responses (McLoughlin et al., 2013). The SbSLSP transgenic plants maintained higher transcript levels of phosphoinositide-specific phospholipase C1 (PLC1) (Figure 7D), suggesting a role of SbSLSP in the salinity and drought stress tolerance through a phosphatidic acid-mediated signaling pathway. The SbSLSP protein contained a clathrin adaptor complex small-chain domain and an $\mathrm{N}$-myristoylation site, and also enhanced the expression of PLC1 in transgenic tobacco, and therefore, it is plausible that SbSLSP is involved in the formation of higher number of CCVs with increased level 

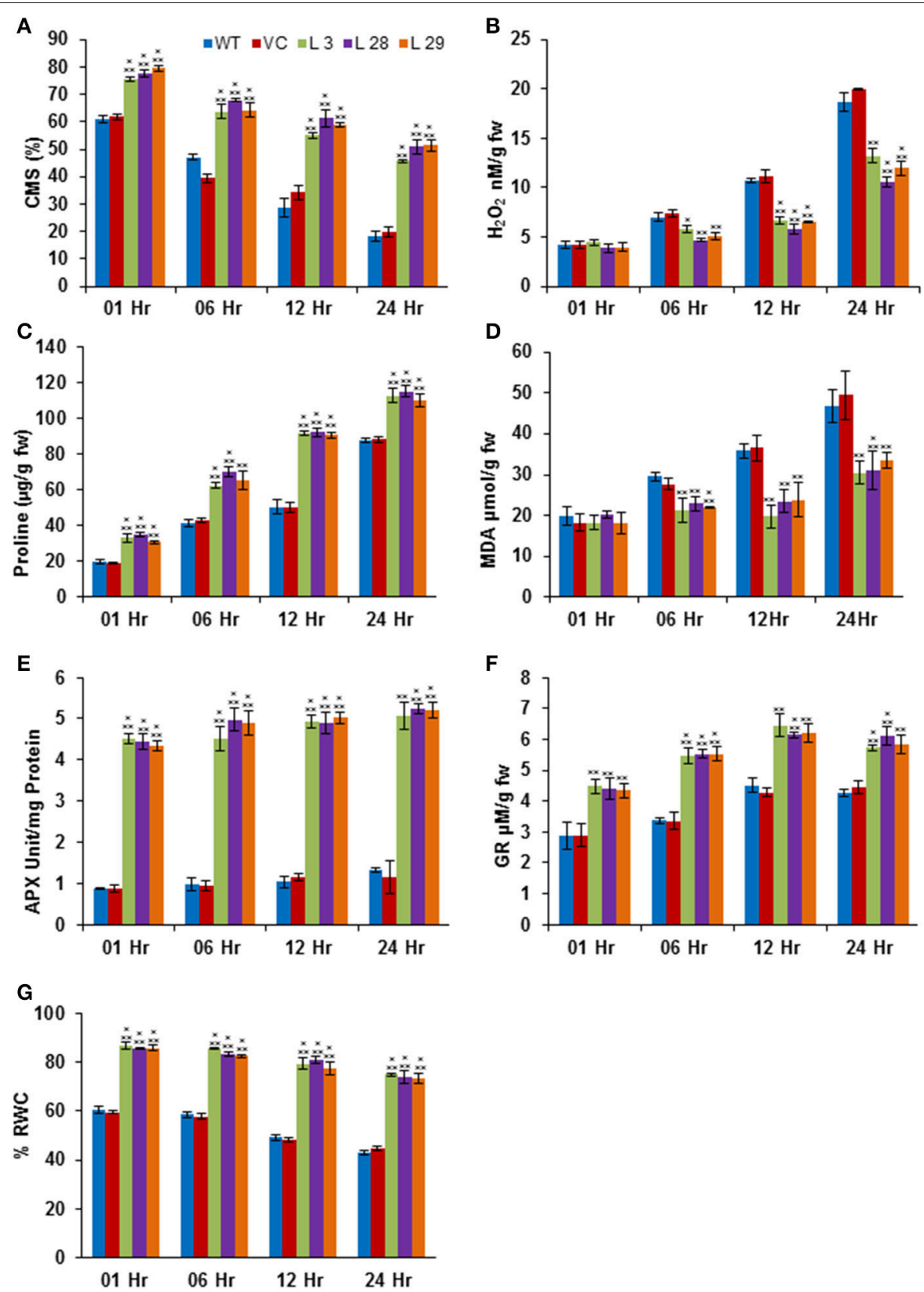

FIGURE 6 | Biochemical and physiological responses of SbSLSP transgenic plants, and VC and WT under drought stress (20\% PEG). (A) Percentage of cell membrane stability. (B) Changes in hydrogen peroxide content. (C) Changes in the level of proline accumulation. (D) Levels of lipid peroxidation expressed in terms of MDA content. (E) Changes in ascorbate peroxidase enzyme activity. (F) Changes in glutathione reductase enzyme activity. (G) Percentage of relative water content. One-way analysis of variance (ANOVA) was used to test the significance between the mean values of control and transgenic plants, and comparison among mean values was performed using ezANOVA (http://www.cabiatl.com/mricro/ezanova/). The difference between control and transgenic lines were statistically significant at ${ }^{*} P<0.05,{ }^{* *} P<0.01$ and ${ }^{* \star *} P<0.001$.

of PtdIns $(4,5) \mathrm{P}_{2}$ and function in phosphatidic acid-mediated salinity and drought-stress adaptation by increasing endocytosis trafficking or signaling during ionic and osmotic stress. It is wellknown that improved trafficking could more efficiently allocate transporters required for water and ion flux. We observed that the transgenic lines overexpressing SbSLSP showed a better water status (Figures 5G, 6G) and lower $\mathrm{Na}^{+}$accumulation with a higher $\mathrm{K}^{+} / \mathrm{Na}^{+}$ratio (Figures $4 \mathrm{~A}-\mathrm{C}$ ), which indicates increased efficiency of transporters.

The SbSLSP-overexpressing tobacco plants promoted seed germination and conferred enhanced tolerance to salinity and osmotic stress (Figures 2, 3). Stressed SbSLSP transgenic plants 
A

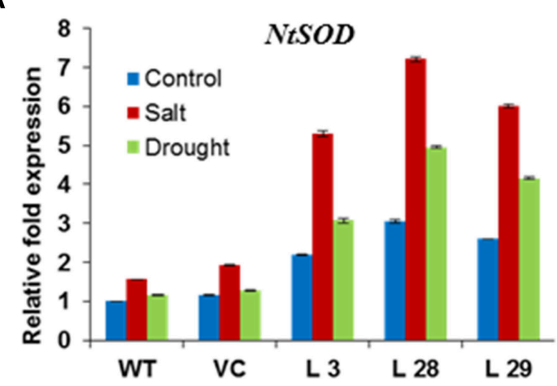

C

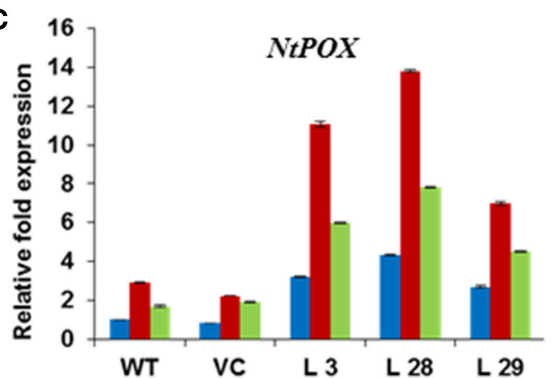

B
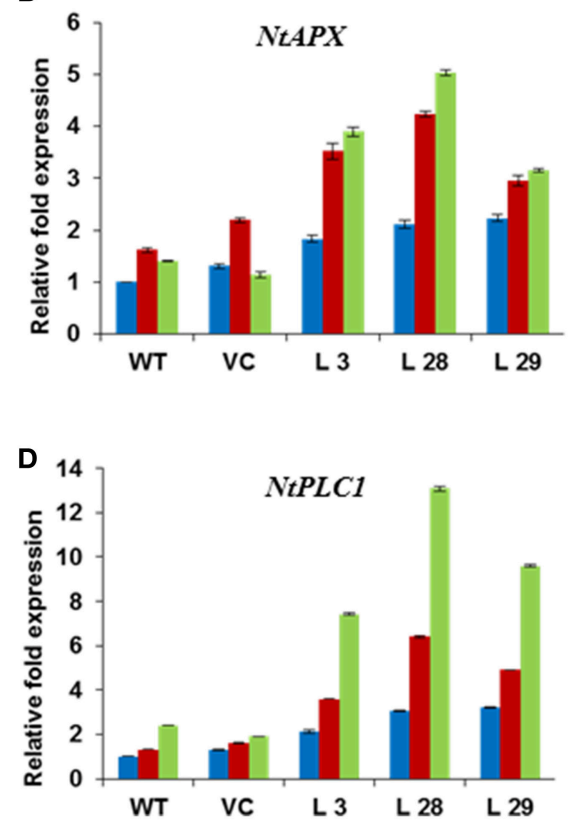

FIGURE 7 | Expression of ROS-scavenging (A) NtSOD, (B) NtAPX, (C) NtPOX, and signaling (D) NtPLC1 genes in SbSLSP transgenic plants, and VC and WT plants under salinity and drought stress using real-time PCR. Actin was used as an internal control. Data represents the mean \pm SD of three replicates.

had longer primary roots compared to control plants, thus higher absorption area, and therefore they are capable of absorbing more water. This may be the reason for higher RWC and biomass synthesis under water-deficit stress. The improved root morphology of SbSLSP transgenic plants permit efficient use of water, and is ultimately contribute to stress tolerance. The higher chlorophyll content, better survival rate and healthier growth of SbSLSP transgenic plants during stress assays corroborate the increased stress tolerance of $S b S L S P$-overexpressing tobacco plants. Similar to our study, Tarte et al. (2015) reported that SNARE gene AtSFT12-overexpressing Arabidopsis plants showed higher salinity and osmotic tolerance and maintained higher RWC compared to WT plants.

Salt stress-induced optimal $\mathrm{Na}^{+}$and $\mathrm{K}^{+}$levels are essential for cellular health in terms of chlorophyll degradation, membrane lipid peroxidation and leaf senescence (Zhu et al., 2001). The SbSLSP-overexpressing tobacco plants accumulated lower $\mathrm{Na}^{+}$and higher $\mathrm{K}^{+}$ions in roots, stems and leaves as compared to WT and VC plants grown in salt stress conditions (Figures 4A-C). SbSLSP-overexpressing tobacco plants also showed a significant improvement in the $\mathrm{K}^{+} / \mathrm{Na}^{+}$ratio (Figure 4C). The above findings suggest that overexpression of SbSLSP acts as a safeguard for the cells from stress-induced injuries and premature senescence. It has been reported that SNAREs protein played crucial role in the regulation of ion transport (Leyman et al., 1999).

In addition to $\mathrm{Na}^{+}$toxicity, salinity, and drought also impose membrane disorganization and generation of ROS (Hasegawa et al., 2000). Various physiological and biochemical parameters have been positively correlated with CMS in stress conditions
(Verslues et al., 2007; Harb et al., 2010; Foyer and Shigeoka, 2011). In the present study, SbSLSP transgenic plants had higher CMS compared to WT and VC plants (Figures 5A, 6A), which is positively correlated with enhanced levels of relative water content and proline. Plants establish their ROS homeostasis in order to minimize oxidative damages to cellular macromolecules (Pitzschke et al., 2009). The SbSLSP-overexpressing lines maintained less ROS in term of MDA and $\mathrm{H}_{2} \mathrm{O}_{2}$ levels in response to salt and drought stresses (Figures 5B,D, 6B,D), which indicates reduced oxidative damage. The ROS detoxifying enzymes activity is improved in plants under numerous biotic and abiotic stresses (Baek et al., 2006; Miller et al., 2010). The transcript levels of ROS-scavenging genes $A P X, P O X$ and $S O D$ were analyzed in overexpressing lines and WT plants. The $S 6 S L S P$-overexpressing lines grown under salinity or drought stress conditions maintained higher transcript levels of these genes relative to WT plants (Figures $\mathbf{7 A - C}$ ). The lower ROS levels in transgenic lines corroborated by the increased transcript levels of ROS-scavenging genes. Bao et al. (2012) also showed that overexpression of SNARE gene OsSYP71 enhanced oxidative stress tolerance of transgenic rice by upregulation of peroxidase.

Based on the findings of our present study, we hypothesized that the enhanced salinity and drought tolerance in the SbSLSP transgenic plants is associated with the higher RWC, less accumulation of ROS, higher accumulation of osmolytes and low $\mathrm{Na}^{+}$content with a better $\mathrm{K}^{+} / \mathrm{Na}^{+}$ratio. By combining the earlier reports and findings of the present study, we proposed a hypothetical model, depicting how SbSLSP may have contributed to achieve enhanced stress tolerance in transgenic tobacco (Figure 8). We hypothesized that constitutive 


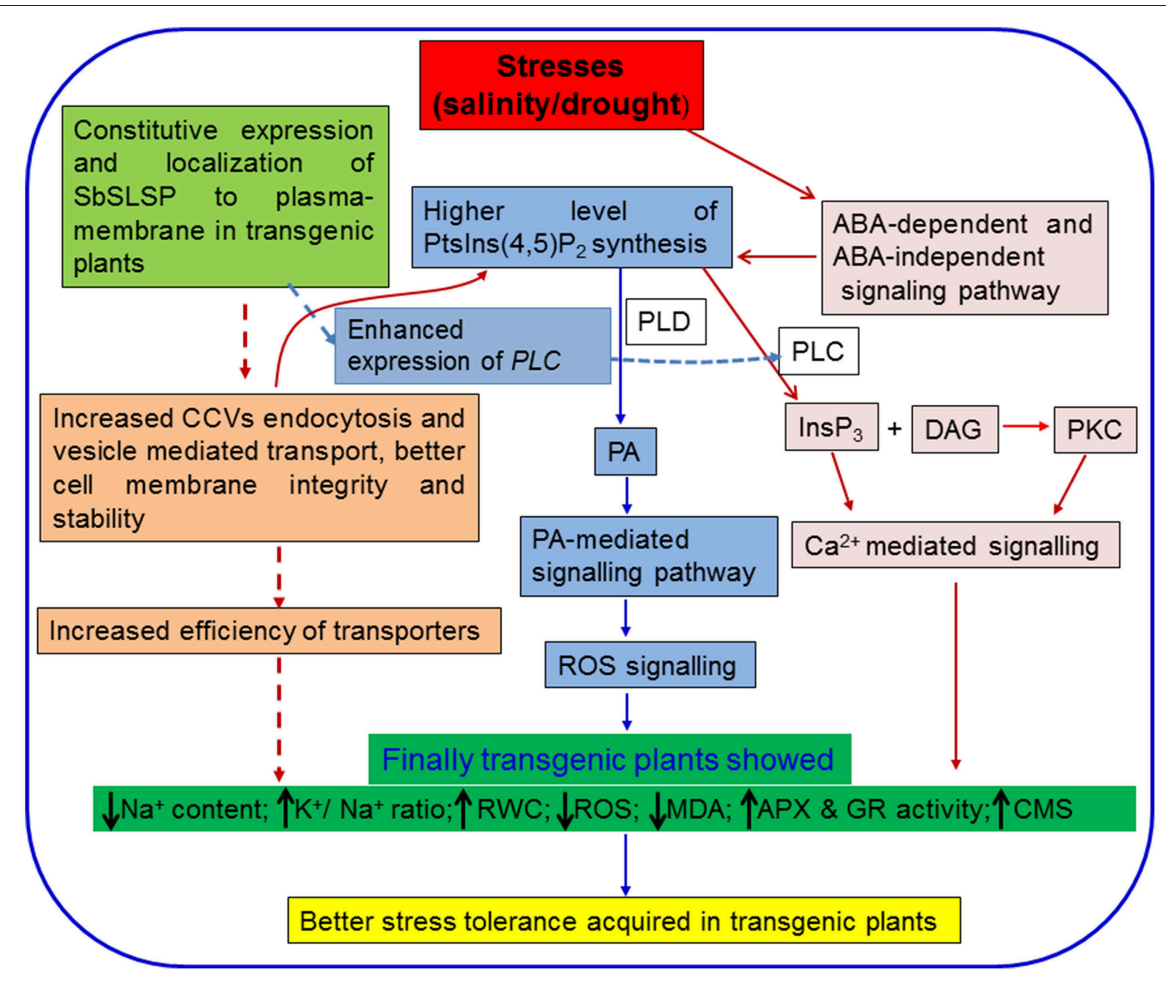

FIGURE 8 | A hypothetical model for the role of SbSLSP in abiotic stress tolerance. The model represents the established signaling cascade under abiotic stresses. The hypothesized plausible role of SbSLSP in stress tolerance was represented by broken lines. Constitutive overexpression of SbSLSP protein may leads to increased clathrin coated vesicles mediated endocytosis leading to higher accumulation of Ptslns $(4,5) \mathrm{P}_{2}$ and then PA mediated stress signaling, which increased the efficiency of transporters and cell membrane stability. The SbSLSP somehow increased the transcript expression of PLC gene which may contribute to the increased $\mathrm{Ca}^{2+}$ mediated signaling pathway. Conclusively all these pathways converge together and results in better abiotic stress tolerance in the transgenic plants. CCVs: Clathrin coated vesicles; PtsIns(4,5) $\mathrm{P}_{2}$ : phosphatidyl-inositol-4,5-bisphosphate; PLC: phospholipase C; PLD: phospholipase D; PA: phosphatidic acid; InsP 3 : inositol-1,4,5-triphosphate; DAG: diacylglycerol; PKC: protein kinase C.

overexpression of SbSLSP protein may leads to increased CCVs mediated endocytosis leading to higher accumulation of PtsIns $(4,5) \mathrm{P}_{2}$ and then PA mediated stress signaling, which increased the efficiency of transporters and cell membrane stability. The SbSLSP also increased the expression of PLC gene, which may contribute to the increased $\mathrm{Ca}^{2+}$ mediated stress signaling pathway. Conclusively all these pathways converse and results in better abiotic stress tolerance in the transgenic plants.

In conclusion, the present study is the first investigation into the function of SbSLSP, a novel salt-inducible gene from halophyte $S$. brachiata. The promoter region not only has several abiotic stress-responsive motifs, but also many of root nodule-specific motifs, which suggests its role in stress tolerance, as well as in nutrient assimilation. Overexpression of SbSLSP resulted in enhanced salinity and drought tolerance, enhanced plant growth, better water status, improved physiological and biochemical parameters, and better regulation of ROS-scavenging genes in response to salt and osmotic stress. Bioengineering of the novel SbSLSP gene may allow development of crop plants with improved stress tolerance, and appears to hold great promise in improving agricultural productivity under high salinity and drought stress.

\section{AUTHOR CONTRIBUTIONS}

Conceived and designed the experiments: NY, PA, and BJ. Performed the experiments: DS, NY, and VT. Analyzed the data: DS, NY, and VT. Wrote the paper: NY and BJ. All authors approved the final manuscript.

\section{ACKNOWLEDGMENTS}

CSIR-CSMCRI Communication No. PRIS-150/2013. This study was supported by the Council of Scientific and Industrial Research (CSIR; http://www.csir.res.in), Government of India, New Delhi [BSC0109: SIMPLE]. NSY and VT are thankful to CSIR for scholarship during the study. The authors are grateful to Arabidopsis Biological Resource Centre, the Ohio State University, USA for providing the pSITE-4CA vector. Mr. Vijay Kumar Singh is acknowledged for his assistance in protein localization experiment.

\section{SUPPLEMENTARY MATERIAL}

The Supplementary Material for this article can be found online at: http://journal.frontiersin.org/article/10.3389/fpls.2016. 00737 


\section{REFERENCES}

Agarwal, P. K., Gupta, K., and Jha, B. (2010). Molecular characterization of the Salicornia brachiata SbMAPKK gene and its expression by abiotic stress. Mol. Biol. Rep. 37, 981-986. doi: 10.1007/s11033-009-9774-1

Agarwal, P. K., and Jha, B. (2010). Transcription factors in plants and ABA dependent and independent abiotic stress signalling. Biol. Plant. 54, 201-212. doi: 10.1007/s10535-010-0038-7

Agarwal, P. K., Yadav, N. S., and Jha, B. (2013). "Role of $\mathrm{Na}^{+} / \mathrm{H}^{+}$antiporters in $\mathrm{Na}^{+}$homeostasis in halophytic plants," in Climate Change and Plant Abiotic Stress Tolerance, eds N. Tuteja and S. S. Gill (Weinheim: Wiley-VCH Verlag $\mathrm{GmbH} \& \mathrm{Co}$. KGaA), 685-704.

Baek, K. H., Skinner, D. Z., Ling, P., and Chen, X. (2006). Molecular structure and organization of the wheat genomic manganese superoxide dismutase gene. Genome 49, 209-218. doi: 10.1139/G05-102

Bao, Y. M., Sun, S. J., Li, M., Li, L., Cao, W. L., Luo, J., et al. (2012). Overexpression of the Qc-SNARE gene OsSYP71 enhances tolerance to oxidative stress and resistance to rice blast in rice (Oryza sativa L.). Gene 504, 238-244. doi: 10.1016/j.gene.2012.05.011

Bates, L. S., Waldern, R., and Teare, I. D. (1973). Rapid determination of free proline for water stress studies. Plant Soil 39, 205-207. doi: 10.1007/BF00018060

Chaturvedi, A. K., Mishra, A., Tiwari, V., and Jha, B. (2012). Cloning and transcript analysis of type 2 metallothionein gene (SbMT-2) from extreme halophyte Salicornia brachiata and its heterologous expression in E. coli. Gene 499, 280-287. doi: 10.1016/j.gene.2012.03.001

Chaturvedi, A. K., Patel, M. K., Mishra, A., Tiwari, V., and Jha, B. (2014). The SbMT-2 gene from a halophyte confers abiotic stress tolerance and modulates ROS scavenging in transgenic tobacco. PLOS ONE 9:e111379. doi: 10.1371/journal.pone.0111379

Chomczynski, P., and Sacchi, N. (1987). Single-step method of RNA isolation by acid guanidinium thiocyanate-phenol-chloroform extraction. Anal. Biochem. 162, 156-159. doi: 10.1016/0003-2697(87)90021-2

El Kasmi, F., Krause, C., Hiller, U., Stierhof, Y. D., Mayer, U., Conner, L., et al. (2013). SNARE complexes of different composition jointly mediate membrane fusion in Arabidopsis cytokinesis. Mol. Biol. Cell 24, 1593-1601. doi: 10.1091/mbc.E13-02-0074

Foyer, C. H., and Shigeoka, S. (2011). Understanding oxidative stress and antioxidant functions to enhance photosynthesis. Plant Physiol. 155, 93-100. doi: $10.1104 /$ pp.110.166181

Garg, B., Jaiswal, J. P., Misra, S., Tripathi, B. N., and Prasad, M. (2012). A comprehensive study on dehydration-induced antioxidative responses during germination of Indian bread wheat (Triticum aestivum L. em Thell) cultivars collected from different agro climatic zones. Physiol. Mol. Biol. Plants 18, 217-228. doi: 10.1007/s12298-012-0117-7

George, S., Parida, A., and Swaminathan, M. S. (2012). "Wheat and rice crops: "Omics" approaches for abiotic stress tolerance," in Improving Crop Resistance to Abiotic Stress, Vol. 1, 2, eds N. Tuteja, S. S. Gill, A. F. Tiburcio and R. Tuteja (Weinheim: Wiley-VCH Verlag GmbH \& Co. KGaA), 695-713.

Godfray, H. C. J., Beddington, J. R., Crute, I. R., Haddad, L., Lawrence, D., Muir, J. F., et al. (2010). Food security: the challenge of feeding 9 billion people. Science 327, 812-818. doi: 10.1126/science. 1185383

Gong, Q., Li, P., Ma, S., Indu Rupassara, S., and Bohnert, H. J. (2005). Salinity stress adaptation competence in the extremophile Thellungiella halophila in comparison with its relative Arabidopsis thaliana. Plant J. 44, 826-839. doi: 10.1111/j.1365-313X.2005.02587.x

Gupta, K., Agarwal, P. K., Reddy, M. K., and Jha, B. (2010). SbDREB2A, an A2 type DREB transcription factor from extreme halophyte Salicornia brachiata confers abiotic stress tolerance in Escherichia coli. Plant Cell Rep. 29, 1131-1137. doi: 10.1007/s00299-010-0896-7

Hamaji, K., Nagira, M., Yoshida, K., Ohnishi, M., Oda, Y., Uemura, T., et al. (2009). Dynamic aspects of ion accumulation by vesicle traffic under salt stress in Arabidopsis. Plant Cell Physiol. 50, 2023-2033. doi: 10.1093/pcp/pcp143

Harb, A., Krishnan, A., Ambavaram, M. M. R., and Pereira, A. (2010). Molecular and physiological analysis of drought stress in Arabidopsis reveals early responses leading to acclimation in plant growth. Plant Physiol. 154, 1254-1271. doi: 10.1104/pp.110.161752
Hasegawa, P. M., Bressan, R. A., Zhu, J. K., and Bohnert, H. J. (2000). Plant cellular and molecular responses to high salinity. Annu. Rev. Plant Biol. 51, 463-499. doi: 10.1146/annurev.arplant.51.1.463

Himabindu, Y., Chakradhar, T., Reddy, M. C., Kanygin, A., Redding, K. E., and Chandrasekhar, T. (2016). Salt-tolerant genes from halophytes are potential key players of salt tolerance in glycophytes. Environ. Exp. Bot. 124, 39-63. doi: 10.1016/j.envexpbot.2015.11.010

Horsch, R. B., Fry, J. E., Hoffmann, N. L., Eichholtz, D., Rogers, S. G., and Farley, R. T. (1985). A simple and general method for transferring genes into plants. Science 227, 1229-1231. doi: 10.1126/science.227.4691.1229

Hu, Y. X., Yang, X., Li, X. L., Yu, X. D., and Li, Q. L. (2014). The SlASR gene cloned from the extreme halophyte Suaeda liaotungensis K. enhances abiotic stress tolerance in transgenic Arabidopsis thaliana. Gene 549, 243-251. doi: 10.1016/j.gene.2014.07.071

Huang, X. S., Luo, T., Fu, X. Z., Fan, Q. J., and Liu, J. H. (2011). Cloning and molecular characterization of a mitogen-activated protein kinase gene from Poncirus trifoliata whose ectopic expression confers dehydration/drought tolerance in transgenic tobacco. J. Exp. Bot. 62, 5191-5206. doi: 10.1093/jxb/err229

Jha, A., Joshi, M., Yadav, N. S., Agarwal, P. K., and Jha, B. (2011). Cloning and characterization of the Salicornia brachiata $\mathrm{Na}^{+} / \mathrm{H}^{+}$antiporter gene SbNHX1 and its expression by abiotic stress. Mol. Biol. Rep. 38, 1965-1973. doi: 10.1007/s11033-010-0318-5

Jha, B., Agarwal, P. K., Reddy, P. S., Lal, S., Sopory, S. K., and Reddy, M. K. (2009). Identification of salt-induced genes from Salicornia brachiata, an extreme halophyte through expressed sequence tags analysis. Genes Genet. Syst. 84, 111-120. doi: 10.1266/ggs.84.111

Jha, B., Lal, S., Tiwari, V., Yadav, S. K., and Agarwal, P. K. (2012). The SbASR -1 gene cloned from an extreme halophyte Salicornia brachiata enhances salt tolerance in transgenic tobacco. Mar. Biotechnol. 14, 782-792. doi: 10.1007/s10126-0129442-7

Jha, B., Sharma, A., and Mishra, A. (2011). Expression of SbGSTU (tau class glutathione S-transferase) gene isolated from Salicornia brachiata in tobacco for salt tolerance. Mol. Biol. Rep. 38, 4823-4832. doi: 10.1007/s11033-010-0625-x

Kim, S. J., and Bassham, D. C. (2011). TNO1 is involved in salt tolerance and vacuolar trafficking in Arabidopsis. Plant Physiol. 156, 514-526. doi: 10.1104/pp.110.168963

Koning, S., Ischebeck, T., Lerche, J., Stenzel, I., and Heilmann, I. (2008). Salt-stressinduced association of phosphatidylinositol 4,5-bisphosphate with clathrincoated vesicles in plants. Biochem. J. 415, 387-399. doi: 10.1042/BJ20081306

Leshem, Y., Melamed-Book, N., Cagnac, O., Ronen, G., Nishri, Y., Solomon, M., et al. (2006). Suppression of Arabidopsis vesicle-SNARE expression inhibited fusion of $\mathrm{H}_{2} \mathrm{O}_{2}$-containing vesicles with tonoplast and increased salt tolerance. Proc. Natl. Acad. Sci. U.S.A. 103, 18008-18013. doi: 10.1073/pnas.0604 421103

Leyman, B., Geelen, D., Quintero, F. J., and Blatt, M. R. (1999). A tobacco syntaxin with a role in hormonal control of guard cell ion channels. Science 283, 537-540. doi: 10.1126/science.283.5401.537

Li, W., Wang, D., Jin, T., Chang, Q., Yin, D., Xu, S., et al. (2011). The vacuolar $\mathrm{Na}^{+} / \mathrm{H}^{+}$antiporter gene SsNHX1 from the halophyte Salsola soda confers salt tolerance in transgenic alfalfa (Medicago sativa L.) Plant Mol. Biol. Rep. 29, 278-229. doi: 10.1007/s11105-010-0224-y

Li, X. L., Yang, X., Hu, Y. X., Yu, X. D., and Li, Q. L. (2014). A novel NAC transcription factor from Suaeda liaotungensis K. enhanced transgenic Arabidopsis drought, salt, and cold stress tolerance. Plant Cell Rep. 33, 767-778. doi: 10.1007/s00299-014-1602-y

Lipka, V., Kwon, C., and Panstruga, R. (2007). SNARE-ware: the role of SNAREdomain proteins in plant biology. Annu. Rev. Cell Dev. Biol. 23, 147-174. doi: 10.1146/annurev.cellbio.23.090506.123529

Livak, K. J., and Schmittgen, T. D. (2001). Analysis of relative gene expression data using real- time quantitative PCR and the 2(-Delta Delta C(T)) method. Methods 25, 402-408. doi: 10.1006/meth.2001.1262

Mahajan, S., and Tuteja, N. (2005). Cold, salinity and drought stresses: an overview. Arch. Biochem. Biophys. 444, 139-158. doi: 10.1016/j.abb.2005.10.018

Mazel, A., Leshem, Y., Tiwari, B. S., and Levine, A. (2004). Induction of salt and osmotic stress tolerance by overexpression of an intracellular vesicle trafficking protein AtRab7 (AtRabG3e). Plant Physiol. 134, 118-128. doi: 10.1104/pp.103.025379 
McLoughlin, F., Arisz, S. A., Dekker, H. L., Kramer, G., Koster, D. C. G., Haring, M. A., et al. (2013). Identification of novel candidate phosphatidic acid-binding proteins involved in the salt-stress response of Arabidopsis thaliana roots. Biochem. J. 450, 573-581. doi: 10.1042/BJ20121639

Miller, G., Suzuki, N., Ciftci-Yilmaz, S., and Mittler, R. (2010). Reactive oxygen species homeostasis and signaling during drought and salinity stresses. Plant Cell Environ. 33, 453-457. doi: 10.1111/j.1365-3040.2009.02041.x

Mukherjee, S. P., and Choudhuri, M. A. (1983). Implications of water stressinduced changes in the levels of endogenous ascorbic acid and hydrogen peroxide in Vigna seedlings. Physiol. Plant. 58, 166-170. doi: 10.1111/j.13993054.1983.tb04162.x

Murashige, T., and Skoog, F. (1962). A revised medium for rapid growth and bioassay with tobacco tissue cultures. Physiol. Plant. 15, 473-497. doi: 10.1111/j.1399-3054.1962.tb08052.x

Nakashima, K., Takasaki, H., Mizoi, J., Shinozaki, K., and Yamaguchi-Shinozaki, K. (2012). NAC transcription factors in plant abiotic stress responses. BBA Gene Regul. Mech. 1819, 97-103. doi: 10.1016/j.bbagrm.2011.10.005

Nakatsu, F., and Ohno, H. (2003). Adaptor protein complexes as the key regulators of protein sorting in the post-Golgi network. Cell Struct. Funct. 28, 419-429. doi: $10.1247 / \mathrm{csf} .28 .419$

Pitzschke, A., Djamei, A., Bitton, F., and Hirt, H. (2009). A major role of the MEKK1-MKK1/2-MPK4 pathway in ROS signalling. Mol. Plant 2, 120-137. doi: $10.1093 / \mathrm{mp} / \mathrm{ssn} 079$

Porra, R. J., Thompson, W. A., and Kriedemann, P. E. (1989). Determination of accurate extinction coefficients and simultaneous equations for assaying chlorophylls a and b extracted with four different solvents: verification of the concentration of chlorophyll standards by atomic absorption spectroscopy. Biochim. Biophys. Acta 975, 384-391. doi: 10.1016/S0005-2728(89)80347-0

Shukla, P. S., Agarwal, P. K., and Jha, B. (2012). Improved salinity tolerance of Arachis hypogaea (L.) by the interaction of halotolerant plant-growthpromoting rhizobacteria. J. Plant Growth Regul. 31, 195-206. doi: 10.1007/s00344-011-9231-y

Singh, D., and Jha, B. (2014). The isolation and identification of salt -responsive novel micro RNAs from salicornia brachiata, an extreme halophyte. Plant Biotechnol. Rep. 8, 325-336. doi: 10.1007/s11816-014-0324-5

Staehelin, L. A., and Newcomb, E. H. (2000). "Membrane structure and membranous organelles," in Biochemistry and Molecular Biology of Plants, eds B. B. Buchanan, W. Gruissem, and R. L. Jones (Rockville: American Society of Plant Physiologists), 2-50.

Tarte, V. N., Seok, H. Y., Woo, D. H., Le, D. H., Tran, H. T., Baik, J. W., et al. (2015). Arabidopsis Qc-SNARE gene AtSFT12 is involved in salt and osmotic stress responses and $\mathrm{Na}^{+}$accumulation in vacuoles. Plant Cell Rep. 34, 1127-1138. doi: 10.1007/s00299-015-1771-3

Tiwari, V., Chaturvedi, A. K., Mishra, A., and Jha, B. (2014). The Transcriptional regulatory mechanism of the peroxisomal ascorbate peroxidase (pAPX) gene cloned from an extreme halophyte, Salicornia brachiata. Plant Cell Physiol. 55, 201-217. doi: 10.1093/pcp/pct172

Tiwari, V., Chaturvedi, A. K., Mishra, A., and Jha, B. (2015). Introgression of the SbASR-1 gene cloned from a halophyte Salicornia brachiata enhances salinity and drought endurance in transgenic groundnut (Arachis hypogaea) and acts as a transcription factor. PLoS ONE 10:e0131567. doi: 10.1371/journal.pone.0131567

Tiwari, V., Patel, M. K., Chaturvedi, A. K., Mishra, A., and Jha, B. (2016). Functional characterization of the Tau class glutathione-s-transferases gene (SbGSTU) promoter of Salicornia brachiata under salinity and osmotic stress. PLoS ONE 11:e0148494. doi: 10.1371/journal.pone.0148494

Topfer, R., Matzeit, V., Gronenborn, B., Schell, J., and Steinbiss, H. H. (1987). A set of plant expression vectors for transcriptional and translational fusions. Nucleic Acids Res. 15:5890. doi: 10.1093/nar/15.14.5890
Udawat, P., Jha, R. K., Sinha, D., Mishra, A., and Jha, B. (2016). Overexpression of a cytosolic abiotic stress responsive universal stress protein (SbUSP) mitigates salt and osmotic stress in transgenic tobacco plants. Front. Plant Sci. 7:518. doi: 10.3389/fpls.2016.00518

Udawat, P., Mishra, A., and Jha, B. (2013). Heterologous expression of an uncharacterized universal stress protein gene (SbUSP) from the extreme halophyte, Salicornia brachiata, which confers salt and osmotic tolerance to E. coli. Gene 536, 163-170. doi: 10.1016/j.gene.2013. 11.020

Uemura, T., Ueda, T., and Nakano, A. (2012). The physiological role of SYP4 in the salinity and osmotic stress tolerances. Plant Signal. Behav. 7, 1118-1120. doi: $10.4161 /$ psb.21307

Verslues, P. E., Kim, Y. S., and Zhu, J. K. (2007). Altered ABA, proline and hydrogen peroxide in an Arabidopsis glutamate:glyoxylate aminotransferase mutant. Plant Mol. Biol. 64, 205-217. doi: 10.1007/s11103-007-9145-Z

Wang, C., Jing, R., Mao, X., Chang, X., and Li, A. (2011). TaABC1, a member of the activity of bcl complex protein kinase family from common wheat, confers enhanced tolerance to abiotic stresses in Arabidopsis. J. Exp. Bot. 62, 1299-1311. doi: $10.1093 /$ jxb/erq377

Yadav, N. S., Rashmi, D., Singh, D., Agarwal, P. K., and Jha, B. (2012a). A novel saltinducible gene SbSI-1 from Salicornia brachiata confers salt and desiccation tolerance in E. Coli. Mol. Biol. Rep. 39, 1943-1948. doi: 10.1007/s11033-0110941-9

Yadav, N. S., Shukla, P. S., Jha, A., Agarwal, P. K., and Jha, B. (2012b). The SbSOS1 gene from the extreme halophyte Salicornia brachiata enhances $\mathrm{Na}^{+}$loading in xylem and confers salt tolerance in transgenic tobacco. BMC Plant Biol. 12:188. doi: 10.1186/1471-2229-12-188

Yadav, N. S., Singh, V. K., Singh, D., and Jha, B. (2014). A Novel Gene SbSI2 Encoding nuclear protein from a halophyte confers abiotic stress tolerance in E. coli and Tobacco. PLoS ONE 9:e101926. doi: 10.1371/journal.pone.01 01926

Yamaguchi-Shinozaki, K., and Shinozaki, K. (2006). Transcriptional regulatory networks in cellular responses and tolerance to dehydration and cold stresses. Annu. Rev. Plant. Biol. 57, 781-803. doi: 10.1146/ annurev.arplant.57.032905.105444

Yang, Y., Tang, R.-J., Jiang, C.-M., Li, B., Kang, T., Liu, H., et al. (2015). Overexpression of the PtSOS2 gene improves tolerance to salt stress in transgenic poplar plants. Plant Biotechnol. J. 13, 962-973. doi: 10.1111/pbi. 12335

Zhu, J., Gong, Z., Zhang, C., Song, C. P., Damsz, B., Inan, G., et al. (2002). OSM1/SYP61: a syntaxin protein in Arabidopsis controls abscisic acidmediated and non-abscisic acid-mediated responses to abiotic stress. Plant Cell 14, 3009-3028. doi: 10.1105/tpc.006981

Zhu, J. K. (2002). Salt and drought stress signal transduction in plants. Annu. Rev. Plant Biol. 4, 401-406. doi: 10.1146/annurev.arplant.53.091401.143329

Zhu, N., Jiang, Y., Wang, M., and Ho, C. T. (2001). Cycloartane triterpene saponins from the roots of Cimicifuga foetida. J. Nat. Prod. 64, 627-629. doi: $10.1021 / \mathrm{np} 000483 \mathrm{k}$

Conflict of Interest Statement: The authors declare that the research was conducted in the absence of any commercial or financial relationships that could be construed as a potential conflict of interest.

Copyright (c) 2016 Singh, Yadav, Tiwari, Agarwal and Jha. This is an open-access article distributed under the terms of the Creative Commons Attribution License (CC $B Y)$. The use, distribution or reproduction in other forums is permitted, provided the original author(s) or licensor are credited and that the original publication in this journal is cited, in accordance with accepted academic practice. No use, distribution or reproduction is permitted which does not comply with these terms. 